\title{
BİR FAZLI DGM DOĞRULTUCU İÇİN LCL FİLTRE TASARIMI VE AKTİF GÜÇ KONTROLÜ
}

\author{
${ }^{1}$ Akif KARAFIL ${ }^{(D)},{ }^{2}$ Harun ÖZBAY ${ }^{(1)}$ \\ ${ }^{1} Y a l o v a$ Üniversitesi, Mühendislik Fakültesi, Elektrik Elektronik Mühendisliğgi Bölümü, Yalova, TÜRKIYE \\ ${ }^{2}$ Bandırma Onyedi Eylül Üniversitesi, Mühendislik ve Doğa Bilimleri Fakültesi, Elektrik Mühendisliği \\ Bölümü, Balıkesir, TÜRKIYY \\ ${ }^{1}$ akif.karafil@yalova.edu.tr, ${ }^{2}$ hozbay@bandirma.edu.tr
}

(Geliş/Received: 07.01.2020; Kabul/Accepted in Revised Form: 05.06.2020)

ÖZ: Darbe genişlik modülasyonlu (DGM) doğrultucu devrelerinin, geleneksel diyotlu doğrultucu devrelerine göre en önemli avantajları DA geriliminin ve güç faktörünün ayarlanabilir olmasıdır. Fakat DGM doğrultucu devrelerinde sert anahtarlama durumu oluştuğundan dolayı şebekede yüksek dereceli akım harmonikleri oluşur. Yüksek dereceli bu akım harmoniklerinin azaltılabilmesi için şebeke tarafında bir filtre kullanılması gerekir. Birçok filtre çeşidi bulunmasına rağmen LCL tipi filtre en çok kullanılan filtre tipidir. Bu çalışmada LCL filtre hesabı yapılarak aktif güç kontrollü bir fazlı DGM doğrultucu devresine ait benzetim ve deneysel sonuçlar verilmiştir. Çalışmanın benzetimi PSIM programında yapılmıştır. Sistemde tüm kontrol yazılımı için program içerisindeki C bloğuna kodlar yazılmış ve program tarafından gömülü kod üretilmiştir. Sistem $400 \mathrm{~W}$ aktif güç aktaracak şekilde tasarlanmış olup güç kontrolü için PI, akım kontrolü için PR denetleyici ve şebeke frekans kontrolü için T/4 gecikmeli faz kilitleme döngü (FKD) algoritması kullanılmıştır.

Anahtar Kelimeler: Aktif güç kontrolü, Bir fazlı DGM doğrultucu, LCL filtre

\section{LCL Filter Design and Active Power Control for Single Phase PWM Rectifier}

\begin{abstract}
The most important advantage of pulse width modulated (PWM) rectifier circuits over traditional diode rectifier circuits are the adjustable DC voltage and power factor. However, due to the hard switching condition in the PWM rectifier circuits, high order current harmonics occur in the grid. In order to reduce these high order harmonics, a filter must be used on the grid side. Although there are many filter types, the LCL type filter is the most commonly used filter type. In this study, simulation and experimental results of active power controlled single phase PWM rectifier circuit were presented by calculating LCL filter. The simulation of the study was conducted in the PSIM program. All control software of the system was achieved by writing codes to the $C$ block in the program, and the embedded codes were produced by the program. The system was designed to transfer $400 \mathrm{~W}$ active power and PI was used for power control, PR controller for current control and T/4 delay phase locking loop (PLL) algorithm for grid frequency control.
\end{abstract}

Key Words: Active power control, Single phase PWM rectifier, LCL filter

\section{GİRIŞ (INTRODUCTION)}

Doğrultucular genel olarak geleneksel ve güç faktörünü düzelten doğrultucular olmak üzere ikiye ayrılır. Geleneksel doğrultucularda diyot ve tristör gibi anahtar elemanları kullanılarak kontrolsüz ve kontrollü doğrultma işlemi gerçekleştirilir. Bu doğrultucuların en önemli dezavantajları güç faktörünün 
düşük olması ve şebeke akımının harmonik bileşenler içermesidir. Bu durum kayıpları arttırırken şebekenin aşırı yüklenmesine neden olur. Bu problemlerin giderilmesi için tek veya çift yönlü güç faktörünü düzelten doğrultucular kullanılmaktadır. Güç faktörünü düzelten doğrultucu devrelerinde MOSFET, IGBT, GTO gibi anahtar elemanları kullanılarak hem güç faktörü yükseltilir hem de şebeke akımının harmonikleri azaltılabilir. Aynı zamanda devrenin DA çıkış gerilimi de ayarlanabilmektedir (Singh ve diğ., 2003; Mansouri ve diğ., 2013).

Güç faktörünü düzelten doğrultucular arasında yer alan bir fazlı DGM doğrultucu devreleri, DA gerektiren uygulamalarda sıklıkla kullanılmaktadır (Song ve diğ., 2016). Uygulamalar arasında elektrikli demiryolu cer sistemi (Gou ve diğ., 2016), elektrikli araçlar (Wang ve diğ., 2019), kesintisiz güç kaynağ1 (Pichan ve diğ., 2017), batarya enerji depolama sistemleri (Mora ve diğ., 2019) ve rüzgâr türbinleri (Freitas ve diğ., 2016) yer almaktadır. DGM doğrultucularda, güç faktörünün ve DA geriliminin kontrol edilebilmesi önemli bir avantaj oluştururken sert anahtarlama nedeniyle oluşan anahtarlama kayıplarının ve akım harmoniklerinin artması ise bu doğrultucuların en büyük dezavantajıdır. Oluşan tek sayılı akım harmoniklerinin azaltılabilmesi için şebeke tarafında bir filtre kullanılmalıdır. Bu amaçla L, LC ve LCL filtreler kullanılarak harmonikler azaltılabilir. Fakat kullanılacak olan filtrenin harmonikleri etkili bir şekilde azaltabilmesinin yan sıra maliyetinin ucuz, boyutunun küçük ve hafif olması istenir. Tüm bu faktörler düşünülerek LCL tipi filtre ile daha hafif ve düşük boyutlu filtre tasarımının yapılabileceği görülmektedir (Liu ve diğ., 2020; Dannehl ve diğ., 2007; Li ve diğ., 2015).

Bir fazlı DGM doğrultucu devrelerinde DA geriliminin ayarlanabilmesi ve güç faktörünün kontrol edilebilmesi için akım ve güç kontrolünün yapılması gerekir. Literatürde akım ve güç kontrolünün sağlanabilmesi için birçok kontrol yöntemi bulunmaktadır (Zhang ve diğg., 2019). Bunlar; model öngörülü (Pan ve Zhang, 2017), deadbeat (Malesani ve diğ., 1999), histerezis (Zhigang ve Hui, 2015), tekrarlamalı (Pandove ve diğ., 2016), bulanık mantık (Lakshmi ve diğ., 2018), oransal integral (PI) (Lin ve Yang, 2004) ve oransal rezonans (PR) (Komurcugil ve diğ., 2016) gibi kontrol yöntemleridir. Bu kontrol yöntemleri arasında PI ve PR en sık kullanılan iki yöntemdir (Bielskis ve diğ., 2020; Heredero-Peris ve diğ., 2019). Diğer kontrol yöntemleri sistemde bir zaman gecikmesi oluştururlar (Karafil ve diğ. 2018).

Bir fazlı DGM doğrultucu devrelerinde şebeke ile senkronizasyonun sağlanabilmesi için faz kilitleme döngüsünün (FKD) kullanılması gerekir. FKD algoritması ile şebeke frekansının kontrolü sağlanmaktadır. DGM doğrultma devrelerinde birçok FKD algoritması kullanılmaktadır. T/4 gecikmeli FKD algoritması faz açısının elde edilebilmesi için kullanılabilecek algoritmalardan biridir (Karafil ve Özbay, 2019).

Bu çalışmada, aktif güç kontrollü LCL filtreli PR akım kontrollü bir faz DGM doğrultma devresinin analizi yapılarak benzetim ve deneysel sonuçlar elde edilmiştir. Benzetim çalışması PSIM programında yapılmış olup sistemin tüm kontrol yazılımı program içerisindeki $C$ bloğu içerisine yazılarak devrenin kontrolünde kullanılan TMS320F28335 DSP için gömülü kod üretimi gerçekleştirilmiştir. Sistemin güç kontrolünde PI, akım kontrolünde ise PR denetleyici kullanılmıştır. Şebeke frekansının kontrolünde T/4 gecikmeli FKD algoritması tercih edilmiştir. Çalışmanın bölümleri şu şekilde düzenlenmiştir: Giriş Bölümü'nden sonra ki kısımda LCL filtre tasarımı ve FKD yapısı anlatılmıştır. Diğer bölümde güç kontrolü ve PR akım kontrol yöntemlerinden bahsedilmiştir. Bu konulardan sonra sistemin benzetim ve deneysel sonuçlarına yer verilmiştir. Sonuç kısmında ise elde edilen benzetim ve deneysel sonuçlar yorumlanmiştır.

\section{LCL FILTRE TASARIMI VE FAZ KİLITLEME DÖNGÜSÜNÜN YAPISI (LCL FILTER DESIGN AND STRUCTURE OF PHASE LOCKED LOOP)}

Bir doğrultma devresinden beklenen şebekeden reaktif güç çekmemesi ve şebekeden çekilen akımın harmonik bileşenler içermemesidir. Şebeke akımının harmonik bileşenler içermemesi için şebeke girişinde harmoniklerin süzülmesi amacıyla filtre kullanılır. Doğrultma devrelerinde birçok filtre tipi kullanılmasına rağmen diğer filtre tiplerine göre daha hafif ve düşük boyutlu LCL tipi filtreler daha çok tercih edilmektedir. Harmoniklerin filtrelenmesinin yanı sıra doğrultma devrelerinde şebeke ile senkronizasyonun da sağlanması gerekir. Bu amaçla faz kilitleme döngüsü kullanılmalıdır. FKD 
algoritması ile şebeke frekansında kilitleme işlemi gerçekleştirilerek şebeke frekansının kontrolü sağlanır (Komurcugil ve diğ., 2016; Karafil ve Özbay, 2019).

\section{LCL Filtre Tasarımı (LCL Filter Design)}

Şekil 1'de LCL filtre bağlantılı bir fazlı DGM doğrultucu devresine ait devre şeması gösterilmiştir. LCL filtre, şebeke tarafında $\mathrm{L}_{\S}$ bobininden, doğrultucu tarafında $\mathrm{Ld}_{\mathrm{d}}$ bobininden ve bobinler arasında paralel bağlı $C_{f}$ kapasitöründen oluşmaktadır. Aynı zamanda osilasyonların engellenmesi için $C_{f}$ kapasitörüne seri bağlı bir $R_{s d}$ sönümleme direnci de sistemde yer almaktadır. LCL tipi filtrenin kullanılması ile bobinlerin endüktans değerleri düşürülürken şebekenin akım harmonikleri de azaltılır.

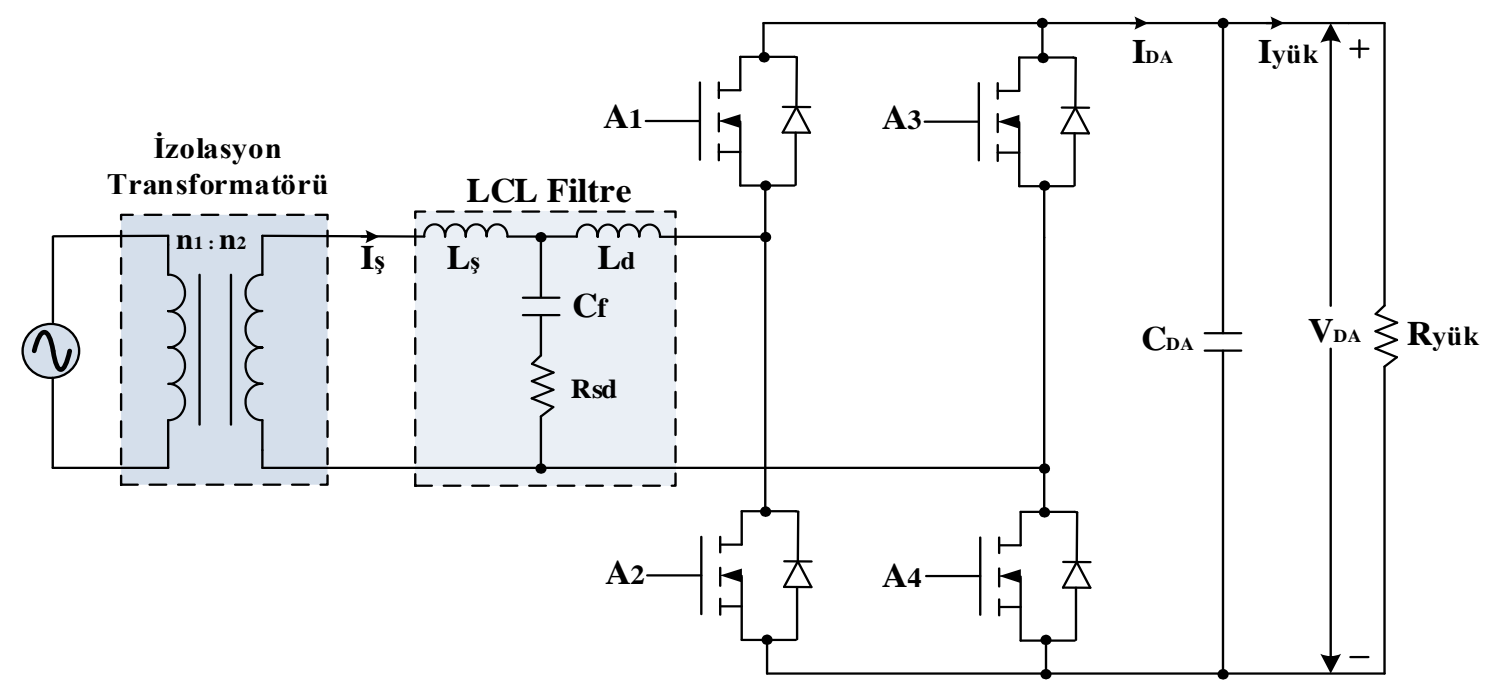

Şekil 1. LCL filtre bağlantılı bir faz DGM doğrultucu devresi

Figure 1. LCL filter connected single phase PWM rectifier circuit

LCL filtre tasarımının yapılabilmesi için öncelikle devre parametrelerinin belirlenmesi gerekir. Çizelge 1'de devreye ait değerler verilmiştir.

Çizelge 1. Doğrultucu devresine ait değişkenlerin değerleri

Table 1. Variable values of rectifier circuit

\begin{tabular}{|l|c|}
\hline \multicolumn{1}{|c|}{ Table 1. Variable values of rectifier circuit } \\
\hline Şebeneke Gerilimi $\left(\mathrm{V}_{\S}\right)$ & Değerleri \\
\hline Çıkış Gücü $\left(\mathrm{P}_{\varsigma}\right)$ & $110 \mathrm{~V}$ \\
\hline DA Gerilimi $\left(\mathrm{V}_{\mathrm{DA}}\right)$ & $400 \mathrm{~W}$ \\
\hline Şebeke Frekansı $(\mathrm{f})$ & $220 \mathrm{~V}$ \\
\hline Anahtarlama Frekansı $\left(\mathrm{f}_{\mathrm{a}}\right)$ & $50 \mathrm{~Hz}$ \\
\hline
\end{tabular}

Doğrultucu tarafındaki bobinin endüktans $\left(\mathrm{Ld}_{\mathrm{d}}\right)$ değeri aşağıdaki eşitlikler ile hesaplanır.

$$
\begin{aligned}
& \Delta I_{L-\max }=a \cdot \frac{P_{c} \sqrt{2}}{V_{s}} \\
& L_{d}=\frac{V_{D A}}{16 \cdot f_{a} \cdot \Delta I_{L-\max }}
\end{aligned}
$$

Burada; (a) akımın dalgalanma oranıdır. Devrenin empedansı ve kapasite değerleri; 


$$
\begin{aligned}
Z_{b} & =\frac{V_{s}^{2}}{P_{c}} \\
C_{b} & =\frac{1}{2 \pi f \cdot Z_{b}}
\end{aligned}
$$

formülleri ile hesaplanır. Filtre kapasitörünün değeri saptanırken $\mathrm{Cb}_{\mathrm{b}}$ değerinin \%5'i alınır. Fakat \% 5 'ten de daha büyük bir değer (k) belirlenebilir.

$$
C_{f}=k \times C_{b}
$$

Şebeke kısmında yer alan bobinin endüktans $\left(\mathrm{L}_{\S}\right)$ değeri saptanırken şebeke ile doğrultucu kısmındaki bobinler arasındaki ilişki katsayısından yararlanılır. Bu katsayı " $\mathrm{r}$ " ile ifade edilir ve $0<\mathrm{r} \leq 1$ aralığında bir değer alır. Bu durumda Lş değeri;

$$
L_{S}=r \cdot L_{d}
$$

formülü ile hesaplanır. Filtrenin rezonans frekansında osilasyona girmemesi için filtre kapasitörüne seri bir sönümleme direnci bağlanır. Sönümleme direncinin $\left(R_{s d}\right)$ değeri, Eşitlik (7)'deki gibi hesaplanır (Karafil, 2020).

$$
R_{s d} \geq \frac{1}{3 \cdot \omega_{r e z} \cdot C_{f}}
$$

Burada; ( $\omega$ rez) rezonans frekansının açısal hızıdır. Rezonans frekansı (frez) ve rezonans frekansının açısal hızı sırasıyla aşağıdaki eşitlikler yardımıyla hesaplanır.

$$
f_{\text {rez }}=\frac{\sqrt{\frac{L_{d}+L_{s}}{L_{d} L_{s} C_{f}}}}{2 \pi}
$$

Rezonans frekansı, anahtarlama frekansından düşüktür. Çünkü LCL filtrenin anahtarlama frekansı içerisinde filtreleme görevini yerine getirebilmesi gerekir. Bu yüzden rezonans frekansı, Eşitlik (10)'daki gösterildiği aralıklarda yer almalıdır (Sun ve diğ., 2009).

$$
10 f \leq f_{\text {rez }} \leq \frac{1}{2} f_{a}
$$

Denklemler yardımıyla $\mathrm{L}_{\mathrm{d}}=3,24 \mathrm{mH}, \mathrm{C}_{\mathrm{f}}=7,892 \mu \mathrm{F}, \mathrm{L}_{\mathrm{s}}=0,972 \mathrm{mH}, \mathrm{R}_{\mathrm{sd}}=4,7 \Omega$ ve frez $=2071,88 \mathrm{~Hz}$ olarak hesap edilmiştir.

\section{Faz Kilitleme Döngüsünün Yapısı (Structure of Phase Locked Loop)}

Şebeke frekansının kontrolü FKD algoritması ile gerçekleştirilir. Temel olarak FKD yapısı bir faz detektörü, bir döngü filtresi ve bir gerilim kontrollü osilatörden oluşmaktadır. Bir fazlı uygulamalarda 
birçok FKD algoritması kullanılır. Bu algoritmalar arasındaki temel fark faz detektörünün düzenlenmesi ve faz hatasının belirlenmesinde sinüzoidal çarpanın uyarlanmasıdır. Bir fazlı sistemlerde kullanılan FKD algoritmaları genel olarak dörde ayrılır. Bunlar; T/4 gecikmeli FKD, ters Park dönüşüm tabanlı FKD, gelişmiş FKD ve ikinci dereceden genelleştirilmiş integratör tabanlı FKD algoritmalarıdır. Bir fazlı uygulamalarda faz açısının elde edilmesinde kullanılan en basit algoritmalardan biri T/4 gecikmeli FKD algoritmasıdır (Ciobotaru ve diğ., 2006; Yang ve Blaabjerg, 2012). Bu çalışmada T/4 gecikmeli FKD algoritması kullanılmıştır. T/4 gecikmeli FKD algoritmasında $\alpha-\beta$ ve d-q eksen takımlarına ihtiyaç vardır. Ortogonal sanal sinyalin ( $\beta$ bileşeni) elde edilebilmesi için $\alpha$ bileşeni $\pi / 2$ kaydırılması gerekir. Bu durumda iki bileşen elde edilir. Bunlardan; $\alpha$ bileşeni reel, $\beta$ bileşeni ise sanal eksendedir. T/4 gecikmeli FKD devresinde $\alpha-\beta$ 'nın Park Dönüşümü ile d-q eksen takımına dönüştürülmesi için Eşitlik (11)'den yararlanılmıştır (Yang ve Blaabjerg, 2012).

$$
\left[\begin{array}{l}
V_{d} \\
V_{q}
\end{array}\right]=\left[\begin{array}{cc}
\cos \omega t & \sin \omega t \\
-\sin \omega t & \cos \omega t
\end{array}\right]\left[\begin{array}{c}
V_{\alpha} \\
V_{\beta}
\end{array}\right]
$$

Şekil 2'de T/4 gecikmeli FKD yapısı gösterilmiştir.

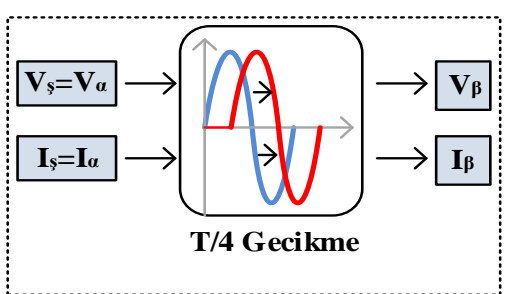

$\alpha-\beta$ Dönüşümü

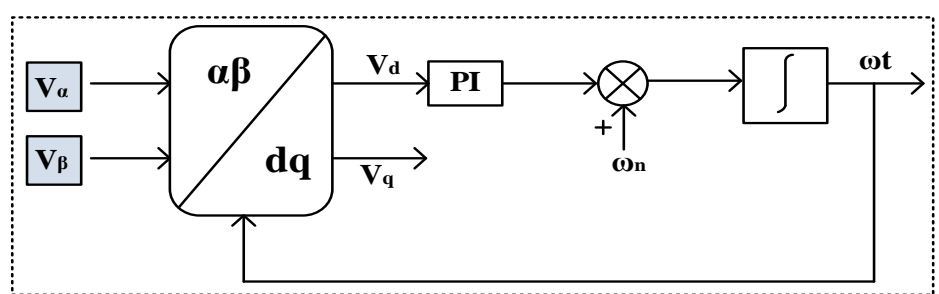

T/4 Gecikmeli FKD

Şekil 2. T/4 gecikmeli FKD yapısı

Figure 2. T/4 delay PLL structure

\section{BİR FAZLI DGM DOĞRULTUCUNUN KONTROLÜ (CONTROL of SINGLE PHASE PWM RECTIFIER)}

Sistemin kontrol blok şeması Şekil 3'te verilmiştir.

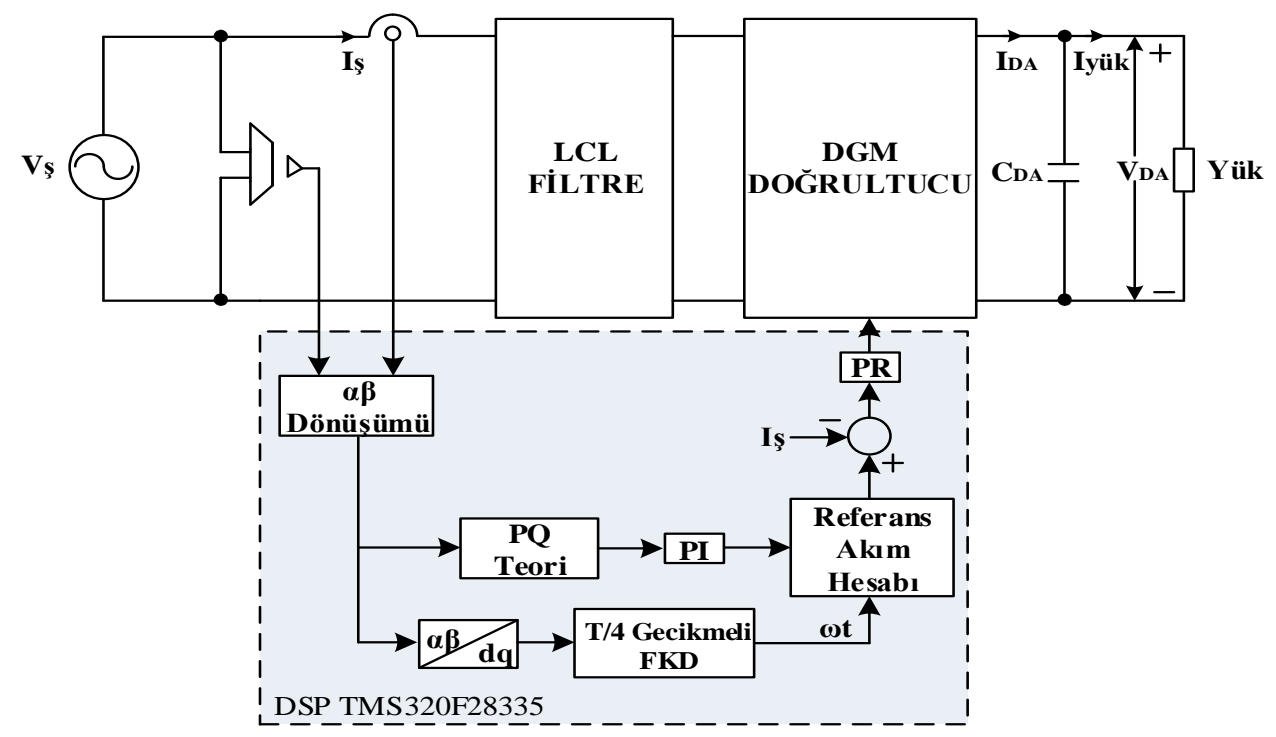

Şekil 3. Bir fazlı DGM doğrultma devresinin kontrol blok şeması

Figure 3. Control block scheme of single phase PWM rectifier circuit 
Aşağıdaki formüller yardımıyla aktif ve reaktif güç (PQ Teori) hesabı yapılmıştır.

$$
\begin{aligned}
& P=\frac{1}{2}\left(V_{\alpha} \cdot I_{\alpha}+V_{\beta} \cdot I_{\beta}\right) \\
& Q=-\frac{1}{2}\left(V_{\alpha} \cdot I_{\beta}+V_{\beta} \cdot I_{\alpha}\right)
\end{aligned}
$$

Elde edilen güç hata değerleri PI denetleyicisinden geçirilmiş ve FKD algoritmasından elde edilen $\omega t$ formüllere dâhil edilerek referans akım hesabı yapılmıştır. Referans akım formülü aşağıdaki eşitlikler kullanılarak bulunmuştur (Karafil ve dĭ̆., 2020).

$$
\begin{aligned}
& \theta=\tan ^{-1}\left(\frac{Q_{r e f}}{P_{r e f}}\right) \\
& I_{S}=\frac{P_{r e f}}{V_{q} \cos \theta} \\
& i_{r e f}=\sqrt{2} I_{S} \sin (\omega t-\theta)
\end{aligned}
$$

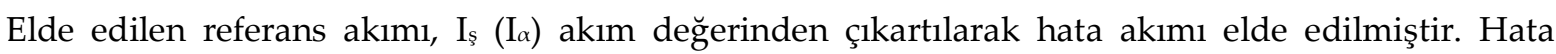
akımı, PR akım denetleyicisinden geçirilerek anahtar sinyalleri oluşturulmuştur. PR denetleyici şebekeye bağlı bir veya üç fazlı sistemlerde kullanılan kontrol yöntemlerinden biridir. PI ve PR, birbirlerine benzeyen ve birçok ortak noktası olan denetleyicilerdir. İki denetleyici arasındaki en önemli fark integrasyonun gerçekleşme biçimidir. PR denetleyicide integral sadece rezonans frekansına yakın frekans değerlerinde işlem yapar. Ayrıca PR denetleyici, PI denetleyicinin bilinen iki önemli dezavantajını ortadan kaldırır. Birincisi sinüzoidal referanslı sıfır kararlı durum hatasıyla takip edilmesi diğeri ise parazitlerin engellenmesidir (Athari ve diğ., 2017; Zhang ve diğ., 2014). Şebekeye bağlı sistemlerin kontrolünde sıfır kararlı durum hatası elde etmek amaciyla genellikle PR denetleyici tercih edilir. PR denetleyicinin uygulanmasında bazı problemler yer alır. İdeal PR denetleyicide sınırsız kazanç harmonik bileşenleri artar. Harmonik bileşenlerinin azaltılması için kullanılan ideal olmayan PR denetleyicinin formülü Eşitlik (17)'de verilmiştir.

$$
G_{n i p r}(s)=K_{p}+\frac{2 K_{i} \omega_{c} s}{s^{2}+2 \omega_{c} s+\omega_{n}^{2}}
$$

Burada, $\omega_{\mathrm{n}}$ şebekenin açısal frekansı, $K_{\mathrm{p}}$ ve $K_{\mathrm{i}}$ değerleri ise sırasıyla oransal ve integral kazanç değerleridir. $\omega_{c}$ ise kesim açısal frekansıdır. İdeal olmayan PR denetleyici de daha düşük kazanç ve bant aralığı mevcuttur (Karafil ve Özbay, 2019; Teodorescu ve diğ., 2006).

Sistemde kullanılan PI ve PR denetleyicilerin parametre değerleri Çizelge 2'de verilmiştir.

Çizelge 2. PI ve PR denetleyicilerin parametre değerleri

\begin{tabular}{|c|c|}
\multicolumn{2}{|c|}{ Table 2. Parameter values of PI and PR controllers } \\
\hline Parametreler & Değerleri \\
\hline FKD algoritmasındaki PI denetleyici için oransal $\left(\mathrm{K}_{\mathrm{p}}\right)$ & 1000 \\
FKD algoritmasındaki PI denetleyici için integral $\left(\mathrm{K}_{\mathrm{i}}\right)$ & 5 \\
\hline Güç kontrolündeki PI denetleyici için oransal $\left(\mathrm{K}_{\mathrm{p}}\right)$ & 5 \\
Güç kontrolündeki PI denetleyici için integral $\left(\mathrm{K}_{\mathrm{i}}\right)$ & 250 \\
\hline Akım kontrolündeki PR denetleyici için oransal $\left(\mathrm{K}_{\mathrm{p}}\right)$ & 2,5 \\
Akım kontrolündeki PR denetleyici için integral $\left(\mathrm{K}_{\mathrm{i}}\right)$ & 400 \\
\hline
\end{tabular}


Doğrusal denetleyiciler olan PI ve PR denetleyicilerin parametre değerleri Ziegler-nichols, kazançfaz marjin yöntemleri kullanılarak bulunmaktadır. Ayrıca optimizasyon algoritmaları kullanılarak da parametre değerleri belirlenebilir (Dos Santos Coelho, 2009). Sistemde kullanılan PI ve PR denetleyicilerin parametre değerleri kazanç-faz marjin yöntemi kullanılarak belirlenmiştir.

\section{BENZETIM VE DENEYSEL SONUÇLARI (SIMULATION AND EXPERIMENTAL RESULTS)}

Bir fazlı DGM doğrultma devresinin PSIM programındaki benzetim ekran görüntüsü Şekil 4'te verilmiştir. DGM doğrultma devresinde yük direnci $120 \Omega$, DA kapasitör değeri $1000 \mu \mathrm{F}$ olarak belirlenmiştir.

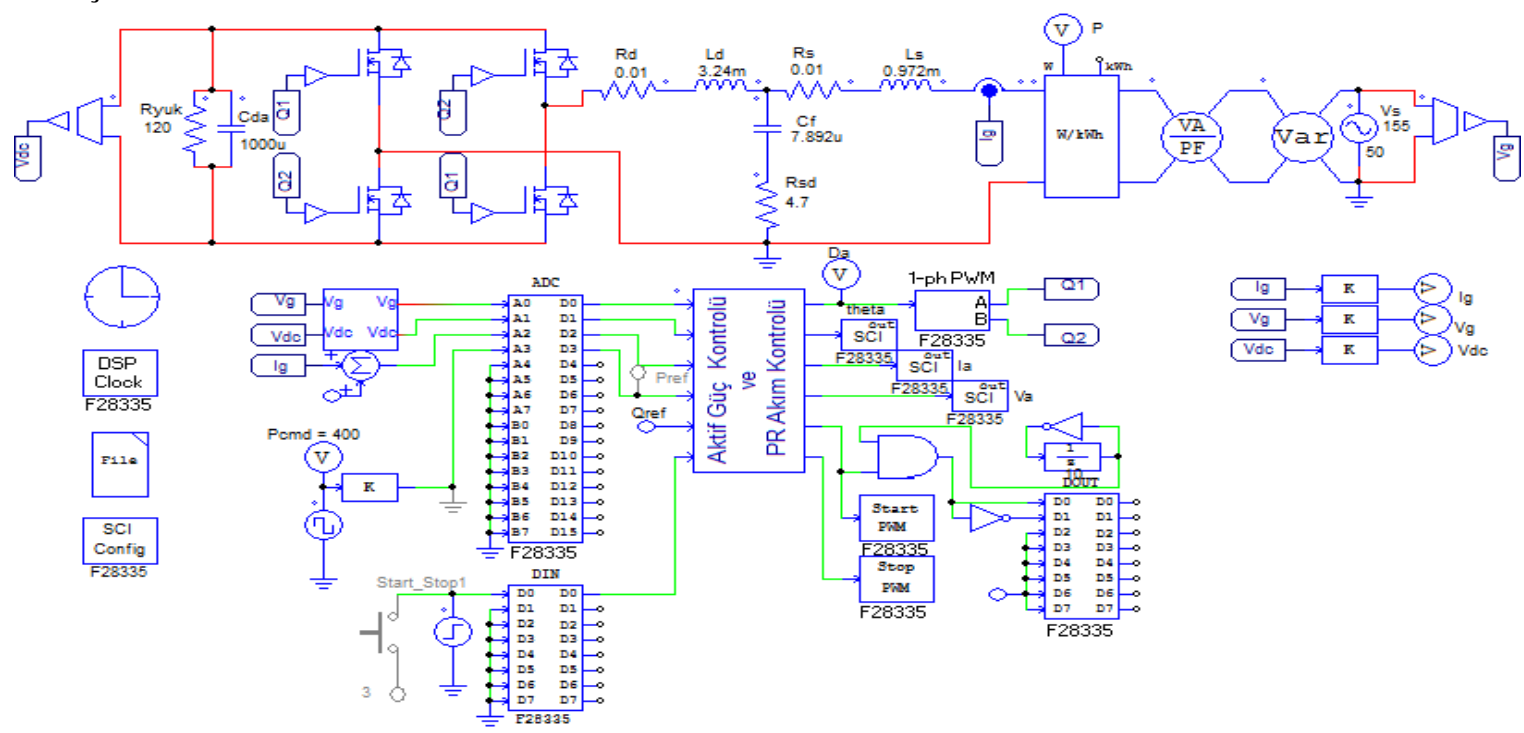

Şekil 4. Bir fazlı DGM doğrultma devresinin benzetimi

Figure 4. Simulation of single phase PWM rectifier circuit

FKD algoritması ile şebeke gerilimi algılanıp sıfır geçiş anları yakalanarak her periyot için şebeke ile senkronize olan açısal hız değerleri üretilmektedir. Şebeke gerilimi ve senkronize olarak üretilen açısal hız değerleri Şekil 5'te gösterilmiştir.

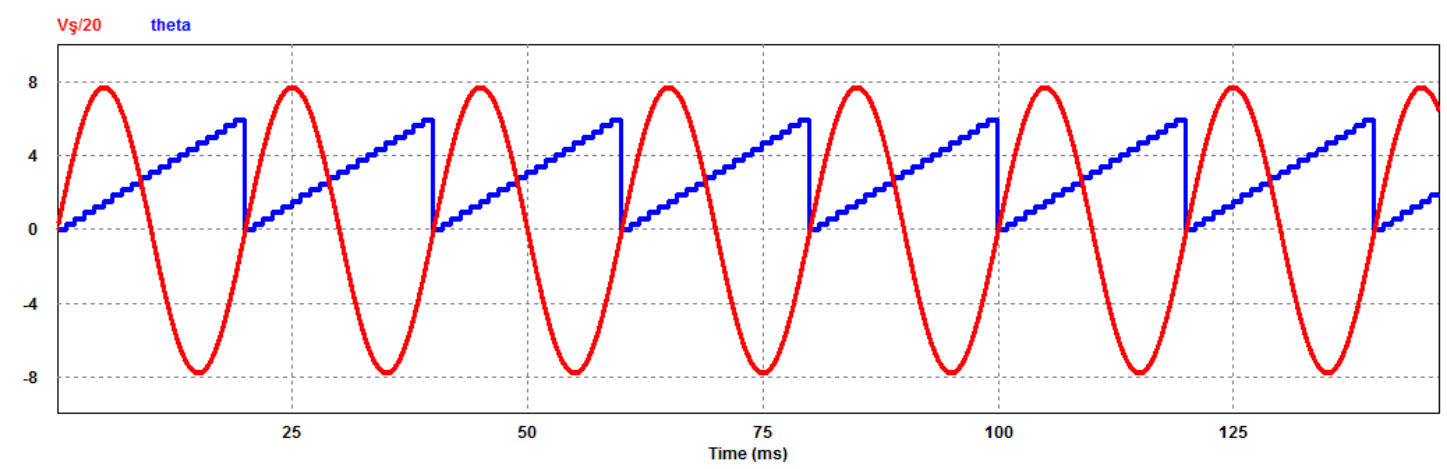

Şekil 5. Şebeke gerilimi ve üretilen açısal hız değerleri

Figure 5. Grid voltage and generated angular speed values

Sistemin aktif gücü $\mathrm{P}=300 \mathrm{~W}$, reaktif gücü $\mathrm{Q}=0$ VAr iken şebekenin akım ve gerilim dalga şekilleri, Şekil 6'da gösterilmiştir. 


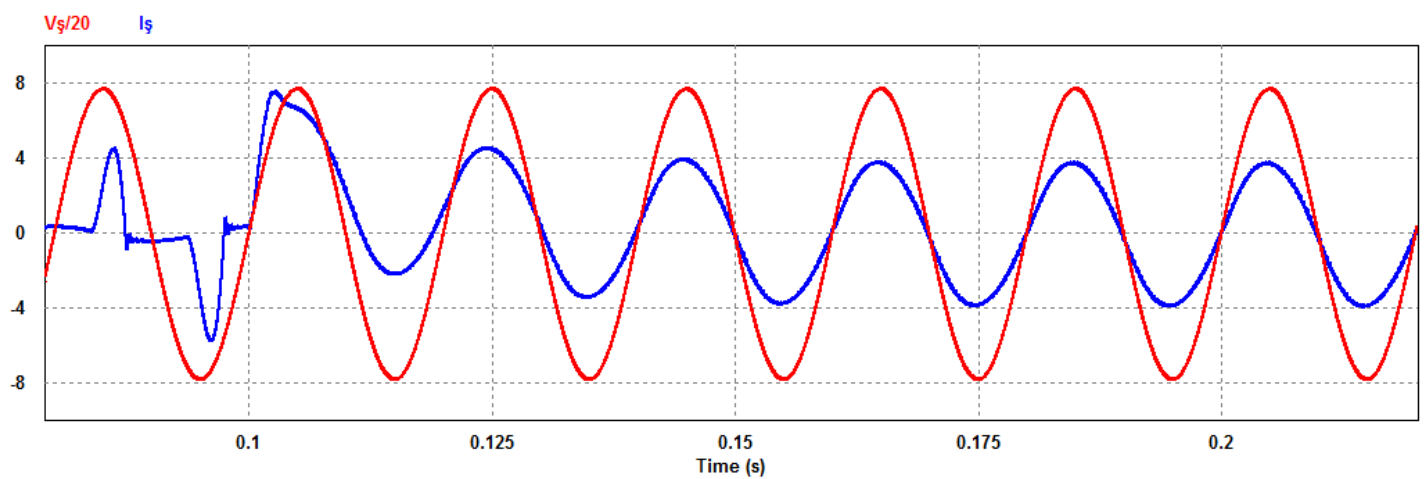

Şekil 6. $300 \mathrm{~W}$ aktif güçteki şebekenin akım ve gerilim değerleri

Figure 6. The current and voltage values of the grid at $300 \mathrm{~W}$ active power

Sistemin $300 \mathrm{~W}$ güçten $400 \mathrm{~W}$ güce geçiş anındaki şebeke akım ve gerilim dalga şekilleri, Şekil 7'de verilmiştir.

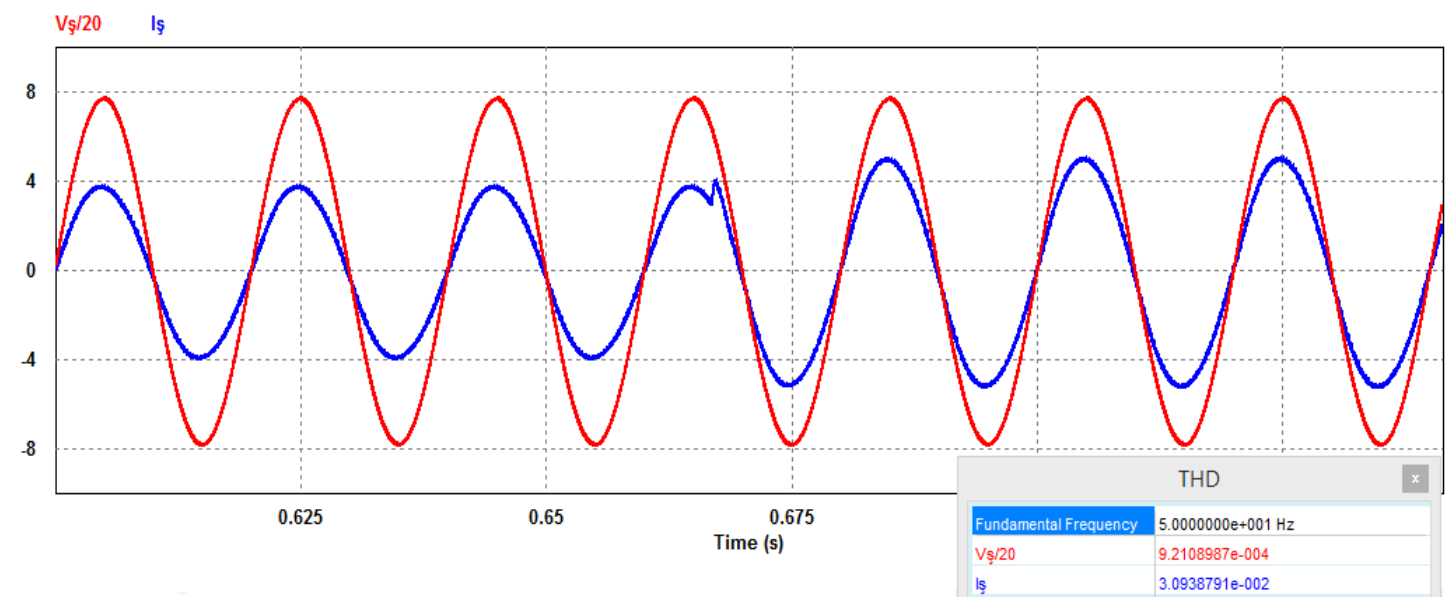

Şekil 7. $300 \mathrm{~W}$ güçten $400 \mathrm{~W}$ güce geçiş anındaki şebekenin akım ve gerilim değerleri Figure 7. The current and voltage values of the grid at $300 \mathrm{~W}$ active power

Şekil 7'den de görüleceği üzere güç geçişleri osilasyonsuz olup şebeke akımının THD değeri de $\% 3,09$ olarak bulunmuştur. Bu durum denetleyicinin dinamik yapısının oldukça iyi olduğunu göstermektedir.

Sistemde 300-400 W aktif güç değerlerinde yük uçlarında oluşan DA gerilim değişimi Şekil 8'de gösterilmiştir.

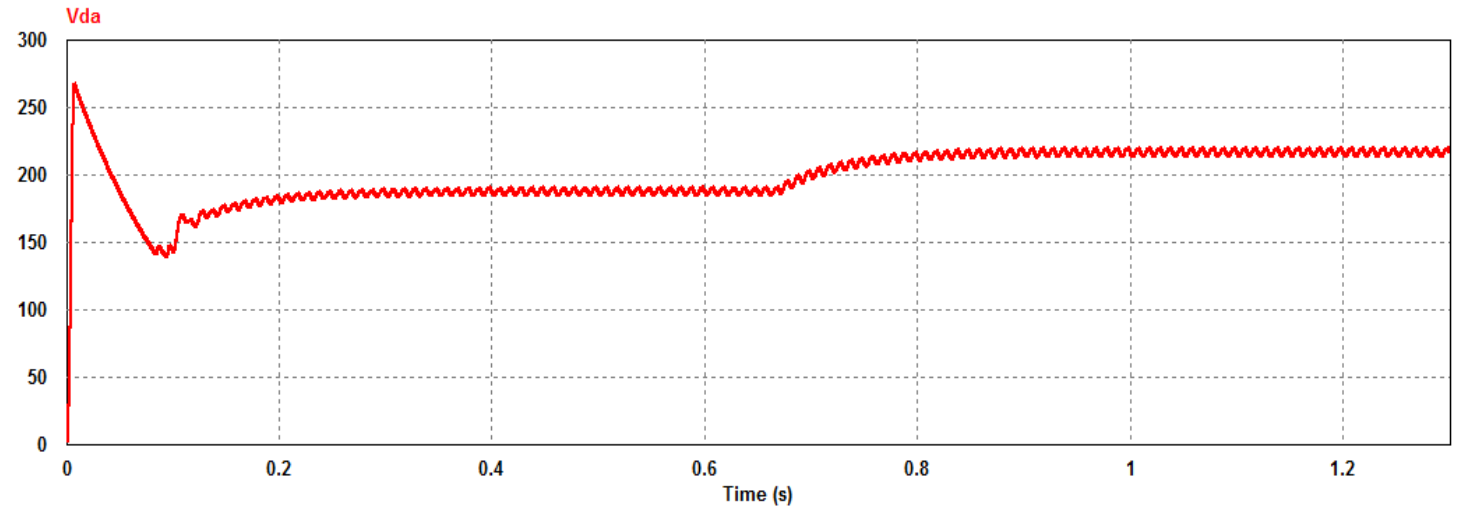

Şekil 8. Yük uçlarındaki DA gerilim değişimi Figure 8. $D C$ voltage change on load terminals 
$400 \mathrm{~W}$ aktif güç değerinde bir fazlı DGM doğrultucu prototip devresi tasarlanmış ve Şekil 9'da tasarlanan devrenin deney düzeneği gösterilmiştir.

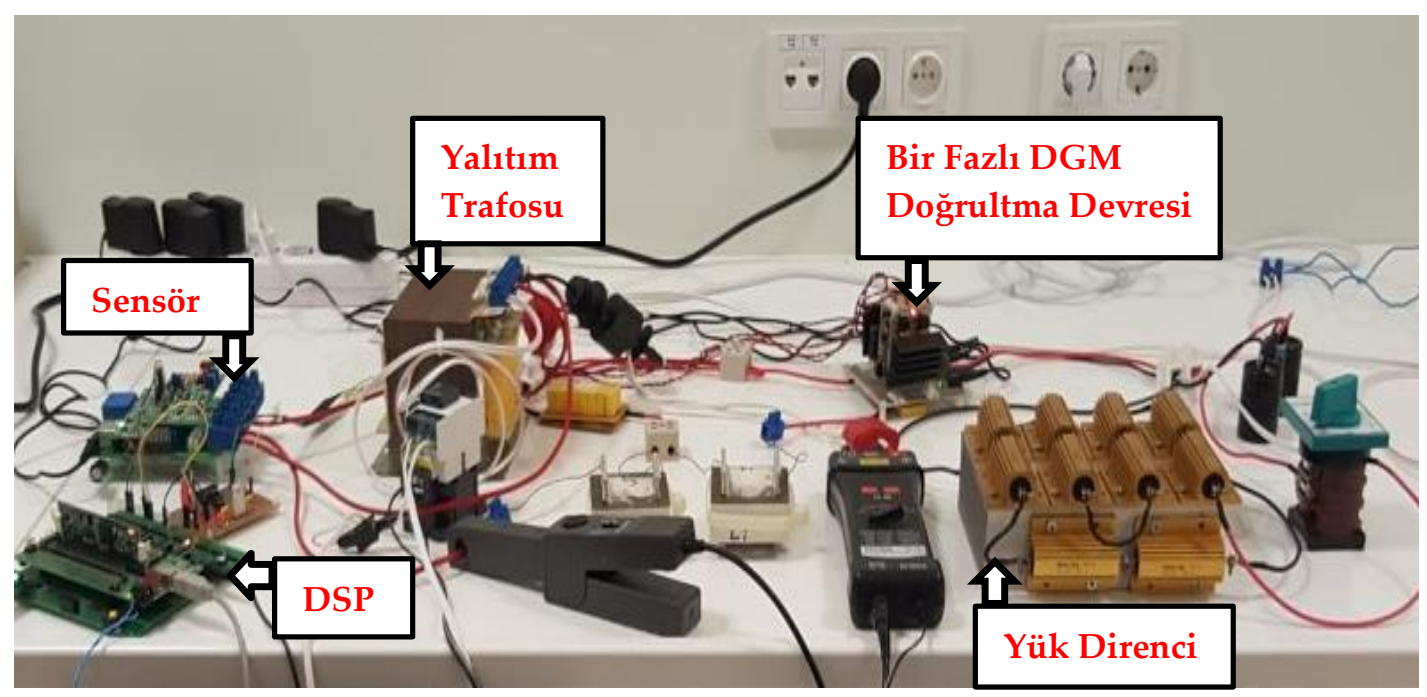

Şekil 9. Bir fazlı DGM doğrultucu devrenin prototipi

Figure 9. The prototype of single phase PWM rectifier circuit

Devre için gerekli olan 110 V'luk şebeke gerilimi yalıtım trafosu kullanılarak sağlanmıştır. Doğrultma devresinde dört adet IRFP460N MOSFET güç anahtarları yer almaktadır. Bir fazlı şebeke akımının ve geriliminin ölçülebilmesi için ACS756SCA-050B ve LV25-P hall etkili sensörler kullanılmıştır. Devrenin kontrolü için bir adet TMS320F28335 sayısal sinyal işlemcisi (DSP) kullanılmıştır. $400 \mathrm{~W}$ aktif güç değerinde test çalışmaları gerçekleştirilmiştir. Devrede reaktif güç kontrolü yapılmadığından $Q$ değeri sıfır olarak alınmıştır. Şekil 10'da şebekeden çekilen akım ve gerilim dalga şekillerinin osiloskop görüntüleri verilmiştir.

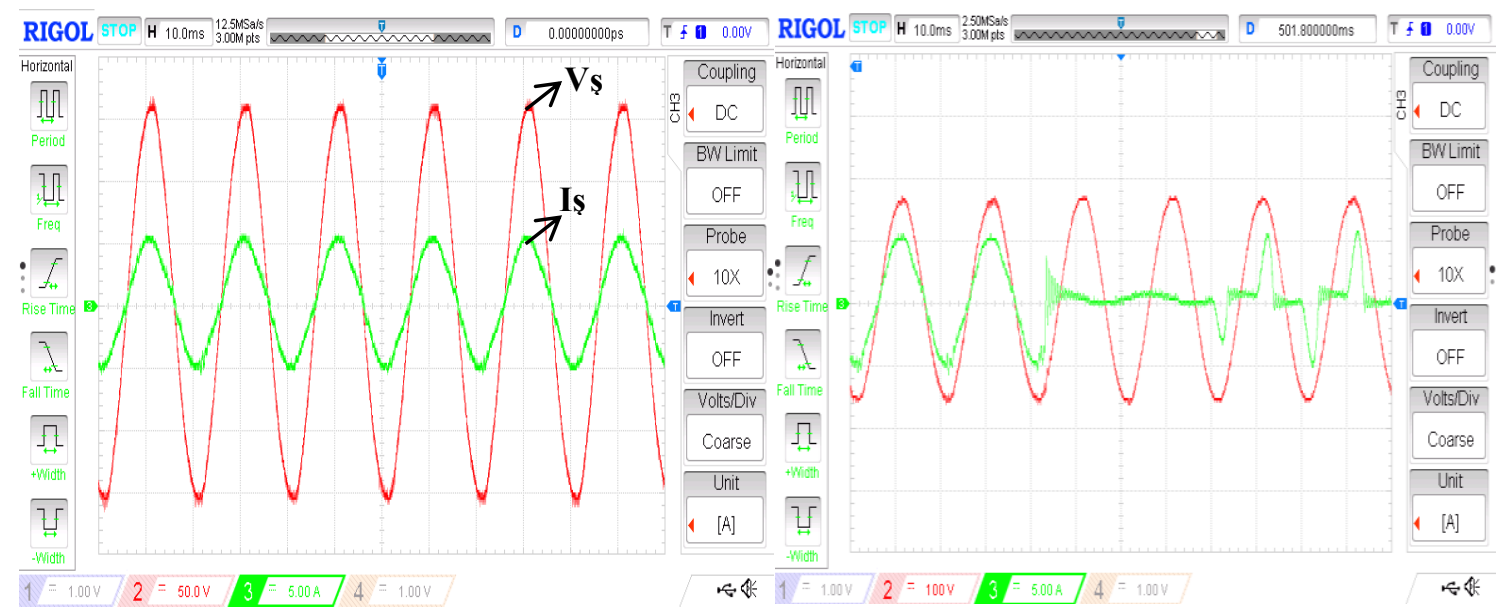

Şekil 10. $400 \mathrm{~W}$ aktif güç değerinde şebekenin akım ve gerilim değerleri

Figure 10. The current and voltage values of the grid at $400 \mathrm{~W}$ active power

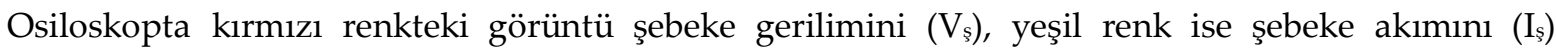
göstermektedir. Şekil 10 'dan da görüleceği üzere aktif güç çekildiğinde akım ile gerilim arasında herhangi bir faz farkı yoktur.

Bir fazlı DGM doğrultucu çıkışındaki $120 \Omega$ yük direnci üzerindeki DA gerilim değişimi Şekil 11'de gösterilmiştir. 


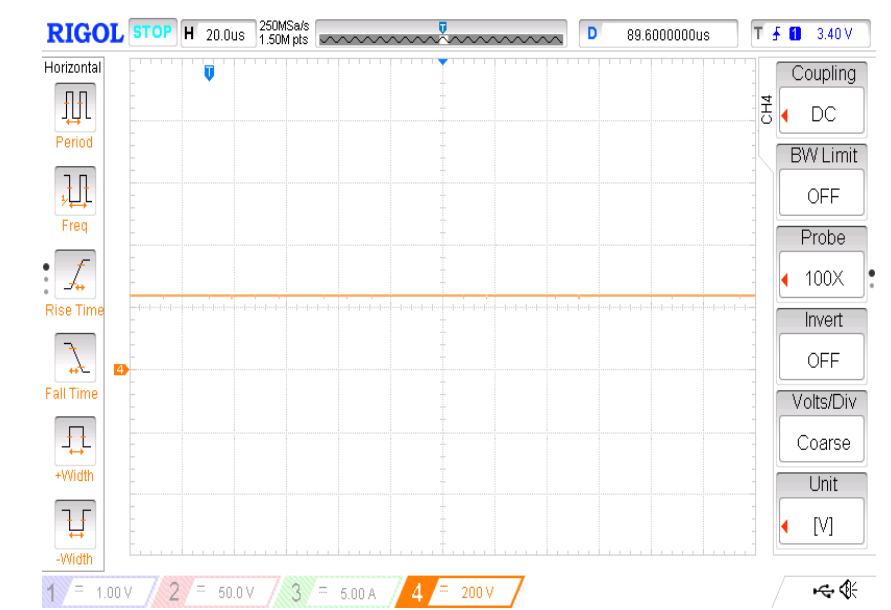

Şekil 11. Bir fazlı DGM doğrultucu devrenin çıkış gerilimi Figure 11. Output voltage of single phase PWM rectifier circuit

Bir fazlı DGM doğrultucunun aktif güç kontrolü hem benzetim hem de deneysel sonuçlar ile gerçekleştirilmiştir. Çalışmada akım kontrolü için PR denetleyici, FKD algoritması için ise T/4 gecikmeli FKD algoritması kullanılmıştır.

\section{SONUÇ ve TARTIŞMALAR (RESULTS and DISCUSSIONS)}

Yapılan bu çalışmada LCL filtreli bir faz DGM doğrultma devresinin aktif güç kontrolünün benzetim ve deneysel sonuçları elde edilmiştir. Güç kontrolü için PI, akım kontrolü için PR denetleyici ve şebeke frekans kontrolü için ise T/4 gecikmeli FKD algoritması kullanılmıştır. Algoritma yazılımları, PSIM programı içerisindeki basitleştirilmiş C bloğunun içerisine yazılmıştır. Sistemde önce LCL filtre hesabı yapılarak tasarım gerçekleştirilmiş daha sonra sistem $400 \mathrm{~W}$ aktif güç değerinde test edilmiştir. Tasarım sonucuna uygun olarak yük uçlarındaki DA gerilim değeri $220 \mathrm{~V}$ ölçülmüştür. Devrenin kontrolü için TMS320F28335 DSP kullanılmıştır. Çalışmada reaktif güç kontrolü yapılmamıştır. İlerleyen çalışmalarda reaktif güç kontrolü de yapılarak aktif-reaktif güç kontrollü DGM doğrultucu devresine ait deneysel sonuçlar sunulacaktır.

\section{KAYNAKLAR (REFERENCES)}

Athari, H., Niroomand, M., Ataei, M., 2017, "Review and Classification of Control Systems in Grid-Tied Inverters", Renewable and Sustainable Energy Reviews, Vol. 72, pp. 1167-1176.

Bielskis, E., Baskys, A., Valiulis, G., 2020, "Controller for the Grid-Connected Microinverter Output Current Tracking", Symmetry, Vol. 12, No. 1, pp. 112.

Ciobotaru, M., Teodorescu, R., Blaabjerg, F., "A New Single-Phase PLL Structure Based on Second Order Generalized Integrator", In 2006 37th IEEE Power Electronics Specialists Conference, Jeju, South Korea, 1-6, 18-22 June 2006.

Dannehl, J., Fuchs, F. W., Hansen, S., "PWM Rectifier with LCL-Filter Using Different Current Control Structures", 2007 European Conference on Power Electronics and Applications, Aalborg, Denmark, 1-10, 2-5 Sept. 2007.

De Freitas, T. R., Menegáz, P. J., Simonetti, D. S., 2016, “Rectifier Topologies for Permanent Magnet Synchronous Generator on Wind Energy Conversion Systems: A Review", Renewable and Sustainable Energy Reviews, Vol. 54, pp. 1334-1344.

Dos Santos Coelho, L., 2009, “Tuning of PID Controller for An Automatic Regulator Voltage System Using Chaotic Optimization Approach", Chaos, Solitons \& Fractals, Vol. 39, No. 4, pp. 1504-1514.

Gou, B., Ge, X., Wang, S., Feng, X., Kuo, J. B., Habetler, T. G., 2016, “An Open-Switch Fault Diagnosis Method for Single-Phase PWM Rectifier Using a Model-Based Approach in High-Speed 
Railway Electrical Traction Drive System", IEEE Transactions on Power Electronics, Vol. 31, No. 5, pp. 3816-3826.

Heredero-Peris, D., Chillón-Antón, C., Sánchez-Sánchez, E., Montesinos-Miracle, D., 2019, “Fractional Proportional-Resonant Current Controllers for Voltage Source Converters", Electric Power Systems Research, Vol. 168, pp. 20-45.

Karafil, A., Özbay, H., Öncü, S., "Bir Fazlı Şebeke Etkileşimli Evirici Tasarımı İçin LCL Filtre Analizi”, Int. Conf. on Data Sci. and Appl. (ICONDATA), Yalova, Türkiye, 554-558, 4-7 Oct. 2018.

Karafil, A., Özbay, H., 2019, "Power Control of Single Phase Active Rectifier", Balkan Journal of Electrical and Computer Engineering, Vol. 7, No. 3, pp. 332-336.

Karafil, A., Özbay, H., Öncü, S., 2020, “Design and Analysis of Single Phase Grid Tied Inverter with PDM MPPT Controlled Converter", IEEE Transactions on Power Electronics, Vol. 35, No. 5, 47564766.

Karafil, A., 2020, "Pasif Seri Sönümleme Direncinin LCL Filtreli Bir Fazlı Şebeke Etkileşimli Evirici Üzerine Etkisi", Pamukkale University Journal of Engineering Sciences, http://doi:10.5505/pajes.2020.99896.

Komurcugil, H., Altin, N., Ozdemir, S., Sefa, I., 2016, “Lyapunov-Function and Proportional-ResonantBased Control Strategy for Single-Phase Grid-Connected VSI with LCL Filter", IEEE Transactions on Industrial Electronics, Vol. 63, No. 5, pp. 2838-2849.

Lakshmi, M. B., Thangasankaran, R., Gnanavadivel, J., Christa, S. J., "Performance Evaluation of Fuzzy Controlled Single Phase PWM Rectifier", 2018 Second International Conference on Electronics, Communication and Aerospace Technology (ICECA), Coimbatore, India, 1974-1979, 29-31 March 2018.

Li, S., Fu, X., Ramezani, M., Sun, Y., Won, H., 2015, “A Novel Direct-Current Vector Control Technique for Single-Phase Inverter with L, LC and LCL Filters", Electric Power Systems Research, Vol. 125, pp. 235-244.

Lin, B. R., Yang, T. Y., 2004, "Single-Phase Half-Bridge Rectifier with Power Factor Correction", IEE Proceedings-Electric Power Applications, Vol. 151, No. 4, pp. 443-450.

Liu, Y., Xu, J., Shuai, Z., Li, Y., Cui, G., Hu, S., Xie, B., 2020, "Passivity-Based Decoupling Control Strategy of Single-Phase LCL-Type VSRs for Harmonics Suppression in Railway Power Systems", International Journal of Electrical Power E Energy Systems, Vol. 117, 105698.

Malesani, L., Mattavelli, P., Buso, S., 1999, "Robust Dead-Beat Current Control for PWM Rectifiers and Active Filters", IEEE Transactions on Industry Applications, Vol. 35, No. 3, pp. 613-620.

Mansouri, M., Kaboli, S. H. A., Selvaraj, J., Rahim, N. A., "A Review of Single Phase Power Factor Correction AC-DC Converters. In 2013 IEEE Conference on Clean Energy and Technology (CEAT), Lankgkawi, Malaysia, 389-394, 18-20 Nov. 2013.

Mora, D., Núñez, C., Visairo, N., Segundo, J., Camargo, E., 2019, “Control for Three-Phase LCL-Filter PWM Rectifier with BESS-Oriented Application”, Energies, Vol. 12, No. 21, 4093.

Pan, L., Zhang, C., 2017, "Model Predictive Control of a Single-Phase PWM Rectifier for Electric Vehicle Charger", Energy Procedia, Vol. 105, pp. 4027-4033.

Pandove, G., Trivedi, A., Singh, M., 2016, "Repetitive Control-Based Single-Phase Bidirectional Rectifier with Enhanced Performance", IET Power Electronics, Vol. 9, No. 5, pp. 1029-1036.

Pichan, M., Ahmad, A. A., Abrishamifar, A., Fazeli, M., "Simple and Efficient Design and Control of the Single Phase PWM Rectifier for UPS Applications", $20178^{\text {th }}$ Power Electronics, Drive Systems $\mathcal{E}$ Technologies Conference (PEDSTC), Mashhad, Iran, 383-388, 14-16 Feb. 2017.

Singh, B., Singh, B. N., Chandra, A., Al-Haddad, K., Pandey, A., Kothari, D. P., 2003, "A Review of Single-Phase Improved Power Quality AC-DC Converters", IEEE Transactions on Industrial Electronics, Vol. 50, No. 5, pp. 962-981.

Song, W., Deng, Z., Wang, S., Feng, X., 2016, “A Simple Model Predictive Power Control Strategy for Single-Phase PWM Converters with Modulation Function Optimization", IEEE Transactions on Power Electronics, Vol. 31, No. 7, pp. 5279-5289. 
Sun, W., Chen, Z., Wu, X., “Intelligent Optimize Design of LCL Filter for Three-Phase Voltage-Source PWM Rectifier", In 2009 IEEE $6^{\text {th }}$ International Power Electronics and Motion Control Conference, Wuhan, China, 970-974, 17-20 May 2009.

Teodorescu, R., Blaabjerg, F., Liserre, M., Loh, P. C., 2006, “Proportional-Resonant Controllers and Filters for Grid-Connected Voltage-Source Converters", IEE Proceedings-Electric Power Applications, Vol. 153, No. 5, pp. 750-762.

Wang, P., Bi, Y., Gao, F., Song, T., Zhang, Y., 2019, “An Improved Deadbeat Control Method for SinglePhase PWM Rectifiers in Charging System for EVs", IEEE Transactions on Vehicular Technology, Vol. 68, No. 10, pp. 9672-9681.

Yang, Y., Blaabjerg, F., "Synchronization in Single-Phase Grid-Connected Photovoltaic Systems under Grid Faults", $20123^{\text {rd }}$ IEEE International Symposium on Power Electronics for Distributed Generation Systems (PEDG), Aalborg, Denmark, 476-482, 25-28 June 2012.

Zhang, N., Tang, H., Yao, C., 2014, "A Systematic Method for Designing A PR Controller and Active Damping of the LCL Filter for Single-Phase Grid-Connected PV Inverters", Energies, Vol. 7, No. 6, pp. 3934-3954.

Zhang, C., Yu, S., Ge, X., 2019, “A Stationary-Frame Current Vector Control Strategy for Single-Phase PWM Rectifier", IEEE Transactions on Vehicular Technology, Vol. 68, No. 3, pp. 2640-2651.

Zhigang, G., Hui, F., "Hysteresis Control with Constant Switching Frequency of Single-Phase PWM Rectifier", $201518^{\text {th }}$ International Conference on Electrical Machines and Systems (ICEMS), Pattaya, Thailand, 559-563, 25-28 Oct. 2015. 